\title{
Association of Polymorphism rs198977 in Human Kallikrein-2 Gene (KLK2) with Susceptibility of Prostate Cancer: A Meta-Analysis
}

\author{
Lishan Wang ${ }^{1,2}$
}

1 Bio-X Institutes, Key Laboratory for the Genetics of Developmental and Neuropsychiatric Disorders (Ministry of Education), Shanghai Jiao Tong University, Shanghai, P.R. China, 2 FengHe (ShangHai) Information Technology Co., Ltd, Shanghai, P.R. China

\begin{abstract}
Objectives: To assess the association of polymorphism rs198977 in the human kallikrein-2 gene (KLK2) and risk of prostate cancer (PCa).

Methods: Two investigators independently searched the PubMed, Elsevier, EMBASE, Web of Science, Wiley Online Library and Chinese National Knowledge Infrastructure (CNKI). Pooled odds ratios (ORs) and 95\% confidence intervals ( $95 \%$ Cls) for rs198977 and PCa were calculated in a fixed-effects model (the Mantel-Haenszel method) and a random-effects model (the DerSimonian and Laird method) when appropriate.
\end{abstract}

Results: Six studies met the inclusion criteria in this meta-analysis, which included 5859 PCa cases and 4867 controls. Overall, rs 198977 was associated with the PCa risk ( $T+C T$ vs. CC, pooled $\mathrm{OR}=1.163,95 \% \mathrm{Cl}=1.076-1.258, \mathrm{P}$-value $<0.0001$ ). When stratified by ethnicity, significant association was observed in Caucasian samples under both allele comparison (T vs. $C$, pooled $\mathrm{OR}=1.152,95 \% \mathrm{Cl}=1.079-1.229$, $\mathrm{P}$-value $<0.0001)$ and dominant model (TT+CT vs. CC, pooled OR $=1.197,95 \%$ $\mathrm{Cl}=1.104-1.297$, P-value $<0.0001$ ). In the overall analysis, a comparably significant increase in the frequency of allele $\mathrm{T}$ for rs 198977 was detected between cases and controls in Caucasian.

Conclusion: This meta-analysis suggests that rs198977 of KLK2 was associated with susceptibility of PCa in Caucasian and the allele T might increase the risk of PCa in Caucasian.

Citation: Wang L. (2013) Association of Polymorphism rs198977 in Human Kallikrein-2 Gene (KLK2) with Susceptibility of Prostate Cancer: A Meta-Analysis. PLOS ONE 8(6): e65651. doi:10.1371/journal.pone.0065651

Editor: Rui Medeiros, IPO, Inst Port Oncology, Portugal

Received December 29, 2012; Accepted April 26, 2013; Published June 18, 2013

Copyright: (c) 2013 Lishan Wang. This is an open-access article distributed under the terms of the Creative Commons Attribution License, which permits unrestricted use, distribution, and reproduction in any medium, provided the original author and source are credited.

Funding: No current external funding sources for this study.

Competing Interests: The author Lishan Wang is employed by Fenghe (ShangHai) Information Technology Co., whose company provided funding towards this study. There are no patents, products in development or marketed products to declare. This does not alter the author's adherence to all the PLOS ONE policies on sharing data and materials.

\section{Introduction}

Prostate cancer (PCa) is the most commonly diagnosed visceral malignancy accounting for more than one-third of all incident cancers and is the second-leading cause of cancer deaths in men in the United States and the western world [1] [2]. Prostate-specific antigen (PSA) has been used for detecting PCa since 1994 [3]. The wide availability of total PSA revolutionized PCa screening and ushered in the PSA era resulting in a decrease of PCa metastasis and death. Several novel blood-based biomarkers, such as human glandular kallikrein 2 (hK2) may also help PCa diagnosis, staging, prognostication, and monitoring [4].

There is considerable evidence for a genetic basis underlying risks for $\mathrm{PCa}$ [5], and it is among the most heritable of the common cancers with a heritability of $42 \%$ estimated from a twin study [6].The kallikrein (KLK) gene family, consisting of 15 genes spanning a region of approximately $300 \mathrm{~kb}$ on $19 \mathrm{q} 13.4$, encodes the largest known cluster of serine-proteases in the human genome [7] [8]. Two of the classical KLK genes, KLK3 and KLK2, that are contiguously located, are expressed almost exclusively in prostate tissue. KLK3 encodes PSA [9], which is produced primarily by prostate epithelium and is widely used as a tumor marker for early detection and monitoring of PCa [10] [11]. KLK2 encodes hK2 that is specific for the prostate and regulates the expression of PSA [12]. They share an $80 \%$ sequence homology and are both primarily expressed in the prostate gland [13]. Using a set of single nucleotide polymorphisms (SNPs) that span the KLK2 region, Nam et al. have demonstrated that KLK2 variants correlate with hK2 serum levels [14]. In addition, Stenman et al. showed that hK2 mRNA expression was significantly higher in PCa tissue compared with benign tissue [15]. 
Table 1. Characteristics of studies included in the meta-analysis.

\begin{tabular}{|c|c|c|c|c|c|c|c|}
\hline Author & Year & Country & Ethnicity & $\begin{array}{l}\text { No.(cases/ } \\
\text { controls) }\end{array}$ & Matching criteria & $\begin{array}{l}\text { Genotyping } \\
\text { methods }\end{array}$ & Ref. \\
\hline Nam, R. K. & 2003 & Canada & Caucasian & $616 / 671$ & Age, race & PCR-RFLP & [17] \\
\hline Chiang,C.H & 2005 & China & Asian & $254 / 168$ & Age, race & PCR-RFLP & [18] \\
\hline Nam, R. K. & 2005 & Canada & Caucasian & $996 / 1092$ & Age, race & PCR-RFLP & [19] \\
\hline Mittal,R. D. & 2007 & India & Asian & $135 / 142$ & Age, race & PCR-RFLP & {$[20]$} \\
\hline Ahn, J. & 2008 & USA & Caucasian & $1172 / 1157$ & Age, race & Sequencing & {$[21]$} \\
\hline Klein,R.J.(a)* & 2010 & Sweden & Caucasian & $1397 / 724$ & Age, race & Sequencing & {$[22]$} \\
\hline Klein,R.J.(b)* & 2010 & Sweden & Caucasian & $1219 / 842$ & Age, race & Sequencing & [22] \\
\hline
\end{tabular}

*Klein's study included two separate groups of samples, data was extracted according to the groups (a and b) as they were independent with each other and analyzed respectively in the initial study. PCR-RFLP: Polymerase chain reaction-restriction fragment length polymorphism.

doi:10.1371/journal.pone.0065651.t001

Moreover, hK2 is expressed at higher level in poorly differentiated cancers and is a likely candidate for prostate carcinogenesis [16].

All of these observations strongly implicate KLK2 as a plausible candidate gene involved in PCa susceptibility. During this decade, a number of studies have assessed the association between polymorphism rs198977 in KLK2 and risk of PCa in different populations; however, the results are inconsistent and inconclusive [17] [18]. Different methodologies have been used, and in particular, samples are collected from all over the world. Therefore, it is not surprising that there has been a lack of replication in different studies. By using all the available published data to increase the statistical power, it was hypothesized that a meta-analysis might allow plausible candidate genes to be excluded and causative genes to be identified with reliability. To confirm whether the polymorphism rs198977 in KLK2 is associated with susceptibility of PCa, we have taken a metaanalysis in which all the published case-control studies are processed.

\section{Materials and Methods}

\section{Literature Search}

Published reports assessing the association between polymorphism of KLK2 and risk of $\mathrm{PCa}$ were collected through a comprehensive search of six databases, including PubMed, Elsevier, EMBASE, Web of Science, Wiley Online Library and Chinese National Knowledge Infrastructure (CNKI). The search terms were as follows: ('KLK2' OR 'kallikrein-2' OR 'hK2') AND ('prostate cancer'). Searching was completed on Nov 1, 2012. Publication date and publication language were not restricted in our search. Meanwhile, reference lists were examined manually to further identify potentially relevant studies. Unpublished reports were not considered. If more than one article was published by the same author using the same case series, the one investigating the most individuals was selected.

\section{Inclusion and Exclusion Criteria}

Abstracts of all citations and retrieved studies were reviewed. Studies meeting the following criteria were included: (1) Using a case-control design; (2) Detecting the relationship between the polymorphism rs198977 and PCa; (3) Providing available genotype data of rs198977. Studies were excluded if one of the following existed: (1) The design was based on family or sibling pairs; (2) The genotype frequency of rs 198977 was not reported; (3) The association of rs198977 with susceptibility of PCa (e.g.

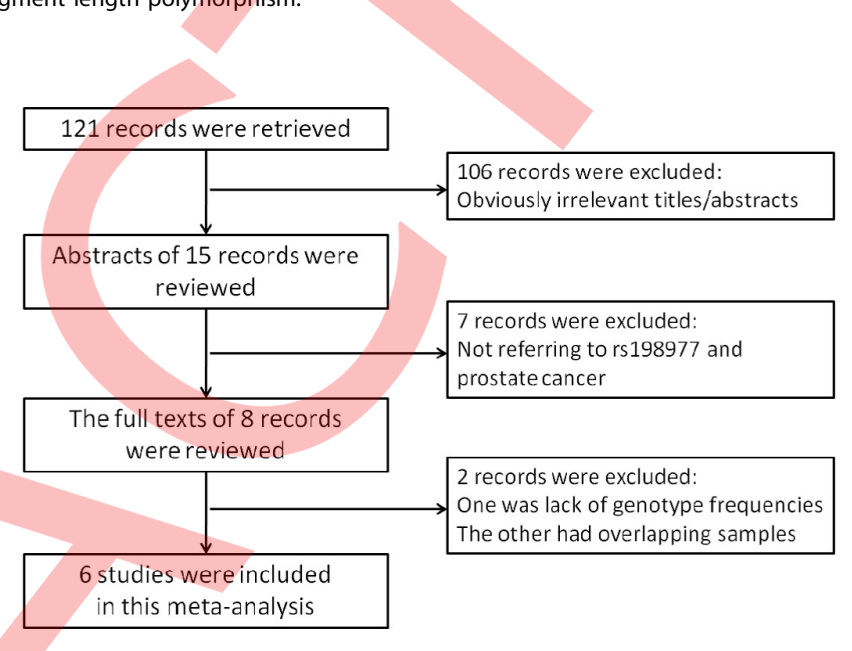

Figure 1. Flow chart of selection of studies and specific reasons for exclusion from the meta-analysis. doi:10.1371/journal.pone.0065651.g001

cancer progression and mortality) was not detected, or (4) There was insufficient information for extraction of data.

\section{Data Extraction}

All data were extracted independently by two reviewers (lishan wang and weidong zang) according to the inclusion criteria listed above. The results were compared and disagreements were discussed and resolved with consensus. Evaluation was based on title and abstract whenever available. Full text articles of potentially relevant studies were obtained and re-evaluated for inclusion. The following characteristics were collected from each study using an Excel data extraction form: first author, year of publication, country of sample, ethnicity, numbers of cases and controls, main background of samples, and genotyping methods (Table 1).

\section{Statistical Analysis}

The statistical analysis was conducted using STATA 11.0 (Stata Corp LP, College Station, TX, United States); P-value $<0.05$ was considered statistically significant. Hardy-Weinberg equilibrium (HWE) in the controls was tested by the chi-square test for goodness of fit, and a $\mathrm{P}$-value $<0.05$ was considered as significant disequilibrium. Pooled odds ratio (ORs) were calculated for allele comparison ( $\mathrm{T}$ vs. G), dominant model (TT+CT vs. GG), and recessive model (TT vs. CC+CT), respectively. The significance of 
Table 2. Genotype frequencies of rs198977 in studies included in the meta-analysis.

\begin{tabular}{|c|c|c|c|c|c|c|c|c|c|}
\hline \multirow[t]{2}{*}{ Author } & \multirow[t]{2}{*}{ Year } & \multicolumn{3}{|c|}{ Case genotype $^{a}$} & \multicolumn{3}{|c|}{ Control genotype $^{b}$} & \multirow[t]{2}{*}{ HWE $^{C}$} & \multirow[t]{2}{*}{ Ref. } \\
\hline & & CC & CT & TT & CC & CT & TT & & \\
\hline Nam, R. K. & 2003 & 315 & 251 & 50 & 394 & 240 & 37 & 0.873 & [17] \\
\hline Chiang,C.H & 2005 & 169 & 83 & 2 & 94 & 52 & 9 & 0.195 & [18] \\
\hline Nam, R. K. & 2005 & 522 & 394 & 80 & 621 & 413 & 58 & 0.782 & [19] \\
\hline Mittal,R. D. & 2007 & 78 & 50 & 7 & 72 & 52 & 18 & 0.211 & {$[20]$} \\
\hline Ahn, J. & 2008 & 621 & 469 & 82 & 660 & 428 & 69 & 0.719 & {$[21]$} \\
\hline Klein,R.J.(a) & 2010 & 788 & 515 & 94 & 443 & 233 & 48 & 0.052 & [22] \\
\hline Klein,R.J.(b) & 2010 & 670 & 460 & 89 & 483 & 287 & 72 & 0.010 & [22] \\
\hline
\end{tabular}

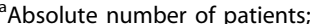

${ }^{b}$ Absolute number of controls;

${ }^{\mathrm{C}} \mathrm{HWE}$ : Hardy-Weinberg equilibrium, it was evaluated using the goodness-of-fit chi-square test. P-values were presented. $\mathrm{P}<0.05$ was considered representative of a departure from HWE.

doi:10.1371/journal.pone.0065651.t002

pooled ORs was determined by Z-test and P-value $<0.05$ was considered as statistically significant.

The OR and 95\% CI were estimated for each study in a random-effects model or in a fixed-effects model. Heterogeneity among studies was examined with the $\chi^{2}$-based Q testing and $I^{2}$ statistics [19]. P-value $<0.1$ was considered significant for the $\chi^{2}$ based $\mathrm{Q}$ testing and $\mathrm{I}^{2}$ was interpreted as the proportion of total variation contributed by between-study variation. If there was a significant heterogeneity ( $\mathrm{P}$-value $<0.1$ ), we selected a randomeffects model (the DerSimonian and Laird method) to pool the data. If not, we selected a fixed-effects model (the Mantel-Haenszel method) to pool the data. Heterogeneity was also quantified using the $I^{2}$ metric $\left(I^{2}<25 \%\right.$, no heterogeneity; $I^{2}=25-50 \%$, moderate heterogeneity; $I^{2}>50 \%$, large or extreme heterogeneity) [25]. Publication bias was examined with funnel plots and with the Egger's tests [20] [21]. If there is evidence of publication bias, the funnel plot is noticeably asymmetric. For the Egger's tests the significance level was set at 0.05 .

\section{Results}

\section{Study Characteristics}

A total of 121 papers were retrieved after the first search, and 115 of these were excluded from the analysis for reasons detailed in Figure 1. Only 6 case-control studies met the inclusion criteria in this meta-analysis, which included $5859 \mathrm{PCa}$ cases and 4867 controls [17] [18]. Characteristics of studies included in the metaanalysis were presented in Tables 1 and 2. The qualities of the studies were considered acceptable for our meta-analysis. We calculated HWE for all six publications and found that only Klein's study [18] was inconsistent with Hardy-Weinberg disequilibrium $(\mathrm{P}$-value $=0.01)$. The flow chart of selection of studies and reasons for exclusion was presented in Figure 1. Studies had been carried out in Canada $(\mathrm{n}=2)$, Sweden $(\mathrm{n}=1)$, USA $(\mathrm{n}=1)$, China $(\mathrm{n}=1)$ and India $(\mathrm{n}=1)$. Four studies [17][22] [23] used Caucasian samples while two studies [24][25] used Asian samples.

Evaluation of rs198977 and Association with PCa

There were six case-control studies [17] [18] which had been performed to study the polymorphism rs198977 and PCa risk. Results of the meta-analysis were shown in Table 3. Overall, when

Table 3. Pooled odds ratio for rs198977 in meta-analyses.

\begin{tabular}{|c|c|c|c|c|c|c|}
\hline Population & Genetic Model & Pooled OR(95\% CI) & P-value ${ }^{a}$ & $\begin{array}{l}\text { P-value }{ }^{b} \text { (Publication } \\
\text { bias) }\end{array}$ & $\begin{array}{l}\text { P-value } \\
\text { (heterogeneity) }\end{array}$ & $I^{2}$ \\
\hline \multirow[t]{3}{*}{ All } & Allele T vs.C & $1.077(0.957-1.212)$ & 0.216 & 0.047 & 0.006 & $67.0 \%$ \\
\hline & Dominant & $1.163(1.076-1.258)$ & $<0.0001$ & 0.050 & 0.143 & $37.4 \%$ \\
\hline & Recessive & $0.993(0.718-1.373)$ & 0.964 & 0.093 & 0.001 & $72.2 \%$ \\
\hline \multirow[t]{3}{*}{ Caucasian } & Allele T vs.C & $1.152(1.079-1.229)$ & $<0.0001$ & 0.328 & 0.319 & $14.9 \%$ \\
\hline & Dominant & $1.197(1.104-1.297)$ & $<0.0001$ & 0.129 & 0.703 & $0.0 \%$ \\
\hline & Recessive & $1.173(0.930-1.480)$ & 0.177 & 0.328 & 0.077 & $52.5 \%$ \\
\hline \multirow[t]{3}{*}{ Asian } & Allele T vs.C & $0.701(0.542-0.906)$ & 0.007 & - & 0.928 & $0.0 \%$ \\
\hline & Dominant & $0.765(0.560-1.045)$ & 0.092 & - & 0.924 & $0.0 \%$ \\
\hline & Recessive & $0.278(0.128-0.600)$ & 0.001 & - & 0.239 & $27.8 \%$ \\
\hline
\end{tabular}

${ }^{a}$ Random-effects model was used when the $p$-value for heterogeneity test $<0.10$, otherwise the fixed-effect model was used.

${ }^{b}$ Egger's test to evaluate publication bias, $\mathrm{P}$-value $<0.05$ is considered statistically significant.

${ }^{c} \mathrm{P}$-value $<0.1$ is considered statistically significant for $\mathrm{Q}$ statistics.

doi:10.1371/journal.pone.0065651.t003 


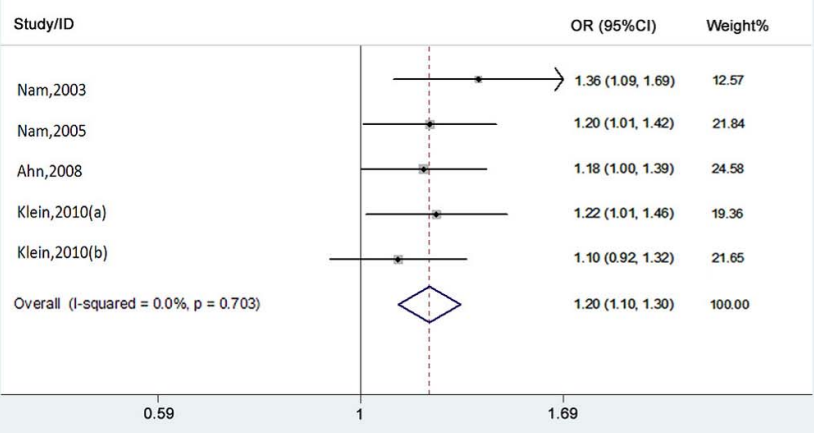

b

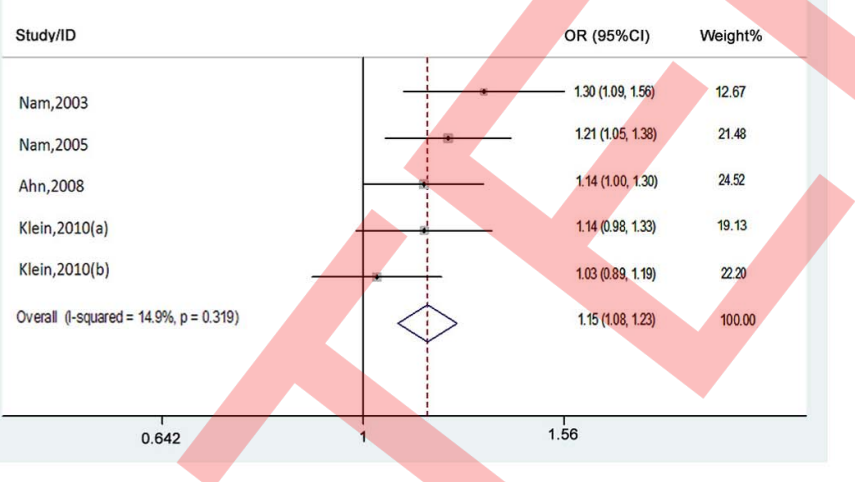

Figure 2. Forest plots of studies with Caucasian samples under dominant model (a) and Allele comparison model (b). doi:10.1371/journal.pone.0065651.g002

all the eligible studies were pooled into the meta-analysis, we found that a significant PCa risk was associated with rs198977 polymorphism in a dominant model (TT+CT vs. CC, pooled $\mathrm{OR}=1.163,95 \% \mathrm{CI}=1.076-1.258, \mathrm{P}$-value $<0.0001$ ); while no significant association was observed in either allele comparison $(\mathrm{T}$ vs. $\mathrm{C}$, pooled $\mathrm{OR}=1.077,95 \% \quad \mathrm{CI}=0.957-1.212$, P-value $=0.216$ ) or recessive model (TT vs. CT+CG, pooled $\mathrm{OR}=0.993,95 \% \mathrm{CI}=0.718-1.373, \mathrm{P}$-value $=0.964)$.

When studies were stratified by ethnicity, significant associations were observed in Caucasian group in both allele comparison ( $\mathrm{T}$ vs. $\mathrm{C}$, pooled $\mathrm{OR}=1.152,95 \% \mathrm{CI}=1.079-1.229, \mathrm{P}$-value $<0.0001)$ and dominant model (TT+CT vs. CC, pooled $\mathrm{OR}=1.197,95 \%$ $\mathrm{CI}=1.104-1.297, \mathrm{P}$-value $<0.0001)$ (Figure 2); while no significant association was observed in Caucasian group in recessive model (TT vs. CT+CG, pooled $\mathrm{OR}=1.173,95 \% \mathrm{CI}=0.930$ $1.480, \mathrm{P}$-value $=0.177)$. In addition, significant associations were observed in Asian group in both allele comparison ( $\mathrm{T}$ vs. $\mathrm{C}$, pooled $\mathrm{OR}=0.701,95 \% \mathrm{CI}=0.542-0.906, \mathrm{P}-\mathrm{value}=0.007)$ and recessive model (TT vs. CT+CG, pooled $\mathrm{OR}=0.278,95 \% \mathrm{CI}=0.128$ $0.600, \mathrm{P}$-value $=0.001)$; while no significant association was

a

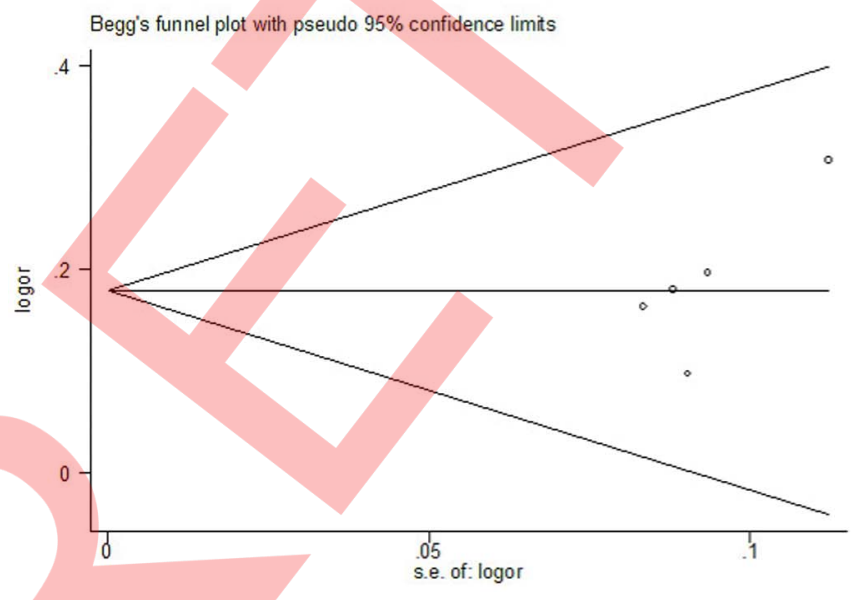

observed in Asian group in dominant model (TT+CT vs. CG, pooled $\mathrm{OR}=0.765,95 \% \mathrm{CI}=0.560-1.045, \mathrm{P}$-value $=0.092$ ).

\section{Sensitivity Analysis}

The influence of a single study on the overall meta-analysis was investigated by omitting one study at a time, and the omission of any study made no significant difference, indicating that our results were statistically reliable.

\section{Evaluation of Heterogeneity}

For all samples, statistically significant heterogeneity was observed under both allele comparison (T vs. C, P-value by $\chi^{2}-$ based $Q$ testing $=0.006$ and $I^{2}=67.0 \%$ ) and recessive model (TT vs. CT+CG, P-value by $\chi^{2}$-based $Q$ testing $=0.001$ and $I^{2}=72.2 \%$ ), but no significant heterogeneity was observed under dominant model (TT+CT vs. CG, P-value by $\chi^{2}$-based $Q$ testing $=0.143$ and $I^{2}=37.4 \%$ ). Then subgroup analysis was carried out. When studies were stratified by ethnicity, no statistically significant heterogeneity was observed in Caucasian under either allele comparison ( $\mathrm{T}$ vs. C, P-value by $\chi^{2}$-based $\mathrm{Q}$ testing $=0.319$ and $I^{2}=14.9 \%$ ) or dominant model (TT+CT vs.

\section{b}

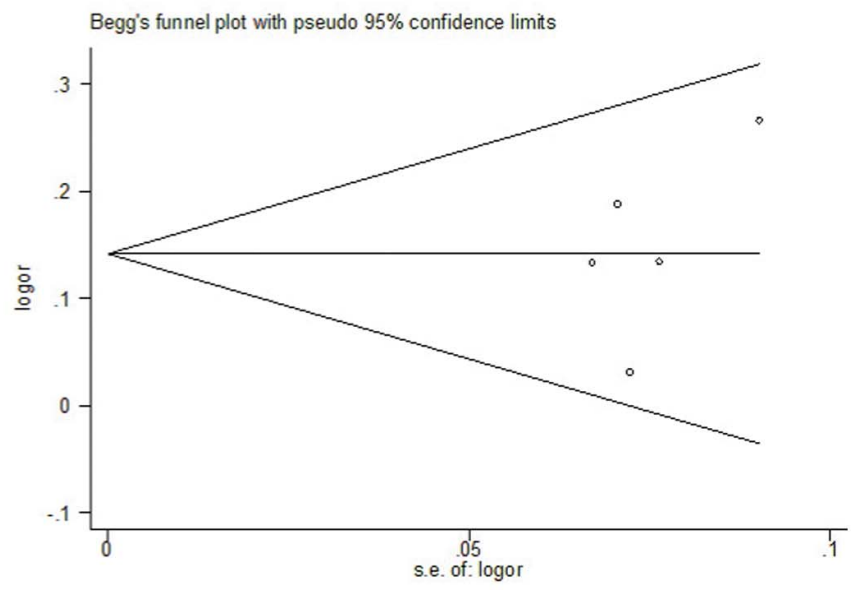

Figure 3. Funnel plots of studies with Caucasian samples under dominant model (a) and Allele comparison model (b). doi:10.1371/journal.pone.0065651.g003 
CG, P-value by $\chi^{2}$-based $Q$ testing $=0.703$ and $I^{2}=0.0 \%$ ), but there was significant heterogeneity under recessive model (TT vs. CT+CC, P-value by $\chi^{2}$-based Q testing $=0.077$ and $\left.I^{2}=52.5 \%\right)$. For Asian, no statistically significant heterogeneity was observed under any model (all P-values by $\chi^{2}$-based Q testing $>0.1$ and $I^{2}<50 \%$ ). Results of heterogeneity were shown in Table 3.

\section{Publication Bias}

Funnel plot and Egger's test were performed to assess the publication bias of the literature. Results publication bias was shown in Table 3. For all samples, publication bias was observed under allele comparison ( $\mathrm{T}$ vs. C, P-value of Egger's test $=0.047$ ). After samples were stratified by ethnicity, no publication bias was observed under any model (all P-value of Egger's test >0.05). Symmetrical funnel plots were obtained for Caucasian (Figure 3), but Funnel plot and Egger's test were not available for Asian samples because of the small sample size.

\section{Discussion}

The data from this meta-analysis showed a significant increase in frequency of genotype TT+CT of rs 198977 polymorphism in patients with $\mathrm{PCa}$ than controls, which suggested that genotype TT+CT might increase the risk of PCa with pooled OR of 1.163. When stratified the samples by their ethnicity, the frequencies of both the allele $\mathrm{T}$ and genotype TT+CT in Caucasian had significant increase in cases than controls with pooled OR of 1.152 and 1.197, respectively; but things became different for Asian, there was a significant decrease in frequency of allele $\mathrm{T}$ in cases than control with pooled OR of 0.701 . Though results from Asian samples indicated allele $\mathrm{C}$ of rs 198977 was the risk factor, it still be under discussion because of the small sample size in our analysis. But the results clearly suggested that allele $\mathrm{T}$ of rs 198977 was a moderate risk factor of $\mathrm{PCa}$ for Caucasian. In addition, Mikolajczyk et al. showed that increased hK2 expression in $\mathrm{PCa}$ tissues could influence cancer biology not only by activating uPA but also by inactivating its primary inhibitor, plasminogen activator inhibitor [26]. And results from transgenic mice demonstrate, with biologically relevant models, that KLK2 is the protease responsible for activating PSA [27]. Variants on KLK2 gene may affect the expression of $\mathrm{hK} 2$, and thus its biological function might be altered. These previous findings support our results and give us possible explanation to the mechanism.

The degree of heterogeneity is one of the major concerns in meta-analysis as non-homogeneous data are liable to result in misleading results. In the present study, the $Q$ testing and $I^{2}$ statistics were carried out to test the significance of heterogeneity. For all samples, significant heterogeneity existed under allele comparison and recessive model. After stratifying the samples according to their ethnicity, the heterogeneity decreased. For

\section{References}

1. Jemal A, Siegel R, Xu J, Ward E (2010) Cancer statistics, 2010. CA: a cancer journal for clinicians 60: 277-300.

2. Siegel R, Ward E, Brawley O, Jemal A (2011) Cancer statistics, 2011: the impact of eliminating socioeconomic and racial disparities on premature cancer deaths. CA: a cancer journal for clinicians 61: 212-236.

3. Nogueira L, Corradi R, Eastham JA (2010) Other biomarkers for detecting prostate cancer. BJU int 105: 166-169.

4. Shariat SF, Semjonow A, Lilja H, Savage C, Vickers AJ et al. (2011) Tumor markers in prostate cancer I: blood-based markers. Acta Oncol 50 Suppl 1: 6175.

5. Langeberg WJ, Isaacs WB, Stanford JL (2007) Genetic etiology of hereditary prostate cancer. Frontiers in bioscience : a journal and virtual library 12: 41014110 .

6. Lichtenstein P, Holm NV, Verkasalo PK, Iliadou A, Kaprio J et al. (2000) Environmental and heritable factors in the causation of cancer-analyses of
Caucasian samples, significant heterogeneity was observed under recessive model, while no statistically significant heterogeneity was observed under any model in Asian samples. The results indicated ethnicity might play an important role in genetic heterogeneity of rs 198977. In other words, there exist heterogeneity of rs 198977 between Caucasian and Asian according to our results.

Publication bias is another important factor affecting the quality of meta-analysis. In order to assess publication bias, funnel plot and Egger's test were performed. Publication bias was observed under allele comparison when all studies were included. After removing Asian samples, no publication bias was observed under any model and symmetrical funnel plots were obtained for Caucasian (all P-value of Egger's test $>0.05$ ) (Table 3 and Figure 3). This indicated that two studies with Asian samples might be responsible for the publication bias, but the reason could not be exactly determined as Funnel plot and Egger's test were not available for only two studies. Moreover, we performed a sensitivity analysis by removing one study each time and rerunning the model to determine the effect on each overall estimate. The estimates changed little, which implied that our results were statistically reliable.

However, there are still some limitations in this meta-analysis. (1) In six studies included for our analysis, two of them are Asian samples occupied only $6.52 \%$ of whole samples, so such results should be interpreted with caution; (2) Because the samples from 5 countries and controls were not uniform, as in most meta-analyses, results should be interpreted with caution; and (3) meta-analysis is retrospective research that is subject to methodological limitations. In order to minimize the bias, we used explicit methods for study selection, data extraction and data analysis. Nevertheless, our results should be interpreted with caution.

This meta-analysis suggests that the polymorphism rs 198977 of KLK2 was associated with susceptibility of prostate cancer in Caucasian and the allele $\mathrm{T}$ might increase the risk of prostate cancer. The pooled ORs in this study suggest that allele $\mathrm{T}$ and genotype TT+CT both have modest but definite genetic effect on prostate cancer in Caucasian. Larger and well-designed studies based on different ethnic groups are needed to confirm our results, especially for Asian samples.

\section{Supporting Information}

\section{Checklist S1 PRISMA checklist. (DOC)}

\section{Author Contributions}

Conceived and designed the experiments: LSW.

cohorts of twins from Sweden, Denmark, and Finland. The New Engl J Med 343: 78-85.

7. Yousef GM, Luo LY, Diamandis EP (1999) Identification of novel human kallikrein-like genes on chromosome 19q13.3-q13.4. Anticancer research 19: 2843-2852.

8. Diamandis EP, Yousef GM, Luo LY, Magklara A, Obiezu CV (2000) The new human kallikrein gene family: implications in carcinogenesis. TEM 11: 54-60.

9. Riegman PH, Vlietstra RJ, van der Korput JA, Romijn JC, Trapman J (1989) Characterization of the prostate-specific antigen gene: a novel human kallikreinlike gene. Biochem biophy res commun 159: 95-102.

10. Catalona WJ, Smith DS, Ratliff TL, Basler JW (1993) Detection of organconfined prostate cancer is increased through prostate-specific antigen-based screening. JAMA 270: 948-954.

11. Catalona WJ, Richie JP, deKernion JB, Ahmann FR, Ratliff TL et al. (1994) Comparison of prostate specific antigen concentration versus prostate specific 
antigen density in the early detection of prostate cancer: receiver operating characteristic curves. The J urol 152: 2031-2036.

12. Partin AW, Catalona WJ, Finlay JA, Darte C, Tindall DJ et al. (1999) Use of human glandular kallikrein 2 for the detection of prostate cancer: preliminary analysis. Urology 54: 839-845.

13. Yousef GM, Diamandis EP (2001) The new human tissue kallikrein gene family: structure, function, and association to disease. Endocrine rev 22: 184-204.

14. Nam RK, Zhang WW, Klotz LH, Trachtenberg J, Jewett MA et al. (2006) Variants of the hK2 protein gene (KLK2) are associated with serum hK2 levels and predict the presence of prostate cancer at biopsy. Clin cancer res 12: 64526458.

15. Lintula S, Stenman J, Bjartell A, Nordling S, Stenman UH (2005) Relative concentrations of hK2/PSA mRNA in benign and malignant prostatic tissue. The Prostate 63: 324-329.

16. Tremblay RR, Deperthes D, Tetu B, Dube JY (1997) Immunohistochemical study suggesting a complementary role of kallikreins hK2 and hK 3 (prostatespecific antigen) in the functional analysis of human prostate tumors. The Am J pathol 150: 455-459.

17. Nam RK, Zhang WW, Trachtenberg J, Diamandis E, Toi A et al. (2003) Single nucleotide polymorphism of the human kallikrein-2 gene highly correlates with serum human kallikrein-2 levels and in combination enhances prostate cancer detection. J clin oncol 21: 2312-2319.

18. Klein RJ, Hallden C, Cronin AM, Ploner A, Wiklund F et al. (2010) Blood biomarker levels to aid discovery of cancer-related single-nucleotide polymorphisms: kallikreins and prostate cancer. Cancer Prev Res (Phila) 3: 611-619.
19. Higgins JP, Thompson SG (2002) Quantifying heterogeneity in a meta-analysis. Statist med 21: 1539-1558.

20. Light RJ, Pillemer DB (1984) Summing up : the science of reviewing research. Harvard University Press, Cambridge, Mass.

21. Egger M, Davey Smith G, Schneider M, Minder C (1997) Bias in meta-analysis detected by a simple, graphical test. BMJ 315: 629-634.

22. Nam RK, Zhang WW, Jewett MA, Trachtenberg J, Klotz LH et al. (2005) The use of genetic markers to determine risk for prostate cancer at prostate biopsy. Clin cancer res 11: 8391-8397.

23. Ahn J, Berndt SI, Wacholder S, Kraft P, Kibel AS et al. (2008) Variation in KLK genes, prostate-specific antigen and risk of prostate cancer. Nature genetics 40: 1032-1034; author reply 1035-1036.

24. Chiang CH, Hong CJ, Chang YH, Chang LS, Chen KK (2005) Human kallikrein-2 gene polymorphism is associated with the occurrence of prostate cancer. J urology 173: 429-432.

25. Mittal RD, Mishra DK, Thangaraj K, Singh R, Mandhani A (2007) Is there an inter-relationship between prostate specific antigen, kallikrein-2 and androgen receptor gene polymorphisms with risk of prostate cancer in north Indian population? Steroids 72: 335-341.

26. Mikolajczyk SD, Millar LS, Kumar A, Saedi MS (1999) Prostatic human kallikrein 2 inactivates and complexes with plasminogen activator inhibitor-1. Int $\mathrm{J}$ cancer 81: 438-442.

27. Williams SA, Xu Y, De Marzo AM, Isaacs JT, Denmeade SR (2010) Prostatespecific antigen (PSA) is activated by KLK2 in prostate cancer ex vivo models and in prostate-targeted PSA/KLK2 double transgenic mice. The Prostate 70: $788-796$.
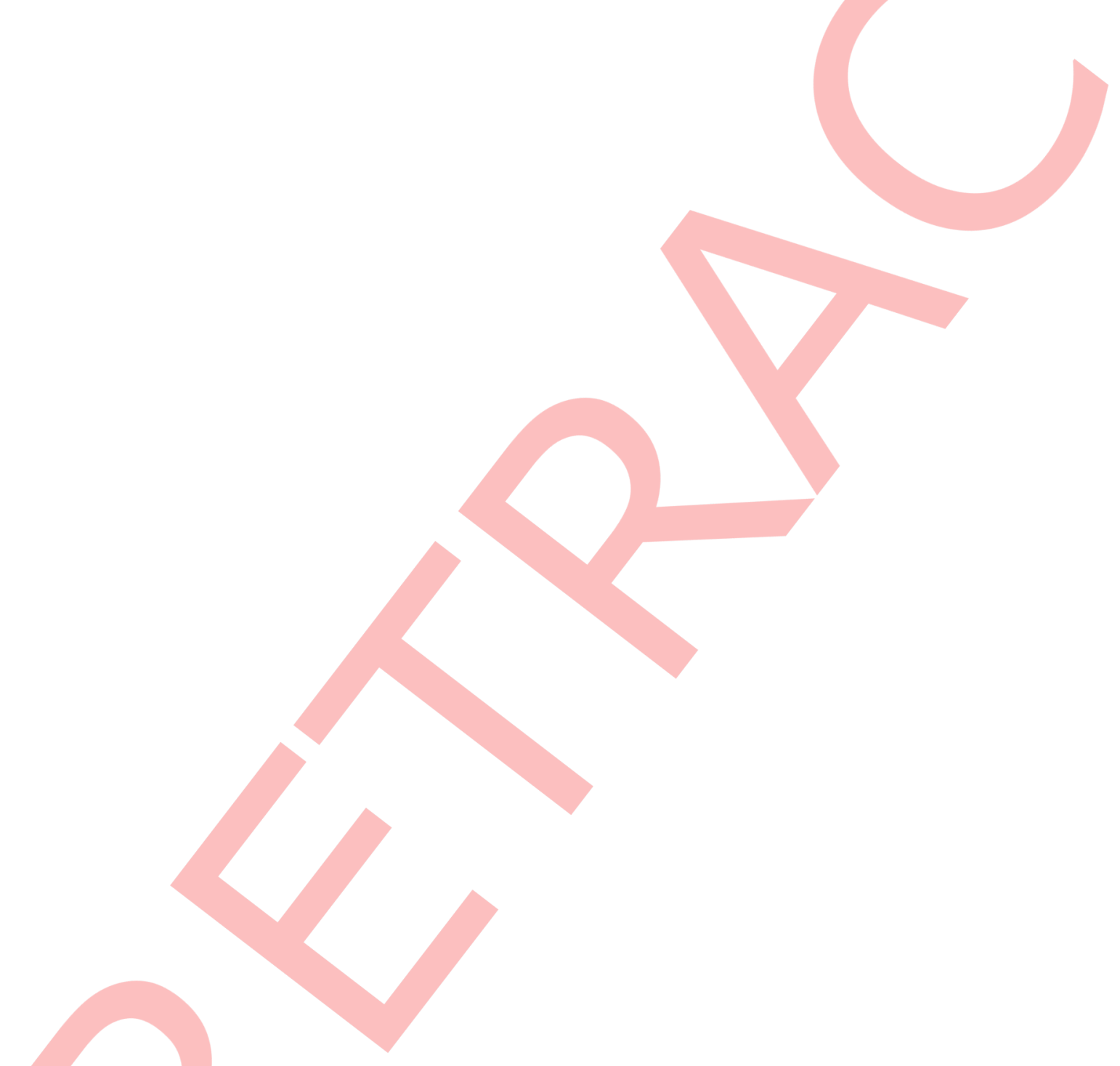